\title{
CLINICAL: OVERVIEW \\ COMMON MENTAL DISORDERS IN PEOPLE LIVING WITH HIV/AIDS
}

\author{
Rita Thom, $M B C h B, D C H, F C P s y c h, P h D$ \\ Division of Psychiatry, Faculty of Health Sciences, University of the Witwatersrand, Johannesburg
}

\begin{abstract}
The term 'common mental disorders' is an overarching term for conditions that affect a significant number of people in the community. These conditions include depression, anxiety and substance use disorders. In contrast, so-called 'severe mental illnesses' (such as schizophrenia or bipolar mood disorder) are conditions that usually require admission for inpatient treatment and tend both to recur and to be chronically debilitating. Nonetheless, common mental disorders result in a considerable burden to the individual, their families, the community and the economy. Many people with these conditions do not present for treatment, or if they do they usually present to primary care facilities, where these diagnoses are often missed. This is unfortunate, since there is good evidence that these disorders can be effectively treated ${ }^{1-2}$ and that much of this treatment can be provided by primary care clinicians. ${ }^{3}$
\end{abstract}

In South Africa, depressive and anxiety disorders are common in the general population. According to the World Health Organization-led South African Stress and Health (SASH) study, the 12-month prevalence of depressive and anxiety disorders (combined) was $12.6 \%{ }^{4}{ }^{4}$ while the lifetime prevalence of these disorders was $25.6 \% .{ }^{5}$ Similarly, the SASH study found a $12-$ month prevalence of $5.8 \%$ and a lifetime prevalence of $13.4 \%$ for substance use disorders. ${ }^{4-5}$

\section{COMMON MENTAL DISORDERS IN HIV-INFECTED} INDIVIDUALS

There is good evidence from international research that the prevalence of depression and anxiety in people living with HIV or AIDS is higher than the prevalence of these disorders in HIV-negative controls. ${ }^{6}$ One meta-analysis suggests that the prevalence is at least double that in the general community. ${ }^{7}$ There is growing evidence that this is true in South Africa and other African countries. ${ }^{8-13}$

The main factors contributing to the increased prevalence of depression and anxiety in individuals living with HIV are:

Biological, resulting from both direct and indirect forms of neurotoxicity due to HIV invasion of the central nervous system and the sequelae of immunocompromise. The areas predominantly affected are the sub-cortical areas of the brain, including those within the temporal lobes, which are the seat of emotional/ mental disorders. ${ }^{14}$ Depressive and anxiety disorders that occur as a result of HIV CNS infection often co- exist with cognitive impairment that is also the result of this infection (see Box 1). Opportunistic conditions such as herpes simplex infection and various malignancies, as well as certain medications used to treat these conditions and antiretroviral medications, can also cause depression or anxiety. ${ }^{15-16}$

Psychological. The prevalence of depression and anxiety in people living with HIV or AIDS is similar to that in people suffering from other serious, chronic and life-threatening medical illnesses. ${ }^{17} \mathrm{~A}$ major factor is the psychological reaction to having such an illness. The individual is faced with the reality of serious illness and possible death at an early age. In many cases there are additional stressors related to the stigma associated with HIV/AIDS and lack of social support for the infected individual (see Box 2).

Primary psychiatric disorder. Some individuals may be particularly vulnerable to depression and anxiety as a result of genetic loading or early childhood adversity. ${ }^{18}$ Some HIV-infected individuals may already have a history of such mental disorder, or the stress of the illness (and the psychosocial consequences) may precipitate a depressive illness and/or an anxiety disorder. In addition, the time of first onset and presentation with primary psychiatric disorders is generally in young adulthood, and this is also the time when most people infected with HIV present for treatment or are diagnosed with the infection. Depressive or anxiety disorders may be completely unrelated to HIV status, or the underlying mental disorder may have contributed to the events associated with acquiring HIV infection (e.g. alcohol abuse and sexual risk taking). 


\begin{tabular}{ll} 
Presentation & Features \\
\hline Somatic & Headache, backache, abdominal pain - may be atypical, or show limited response to \\
& somatic treatments \\
Insomnia & Common presentation; an important depressive symptom \\
Other neuro-vegetative symptoms & Fatigue, weight loss, loss of energy and libido \\
Low mood or depression & May be expressed differently, such as 'stress' or tension \\
Substance abuse & This commonly coexists with depression, and may be a presenting feature
\end{tabular}

Several of the most frequently observed presentations of common mental disorders are listed in Table I. Identifying depression in a medically ill individual can be difficult owing to the overlap in somatic symptoms (e.g. loss of appetite or weight, sleep disturbance, pain, fatigue, poor concentration). It is important to consider mood and affect, particularly if alterations in these are constant, unvarying and more severe than is warranted by the individual's circumstances, as well as anhedonia (loss of pleasure in usually enjoyable activities) and functional impairment caused by the symptoms that are present. It is also suggested that an inclusive approach should be used when considering the criteria for making a diagnosis of depression in a medically ill individual. ${ }^{17}$ The relationship between substance abuse, mood and anxiety disorders and HIV infection is complex, and all these disorders may play a role in increasing the risk of being infected with HIV, as well as contributing to the burden of disease, poor adherence and disease progression.

\section{BOX 1}

Mrs $\mathrm{K}$ is a 50 -year-old woman living in a rural area. Her husband died of AIDS a year ago. She presents at the clinic with severe oral thrush. She is HIV positive and has severe weight loss. She looks depressed and is very tearful, complaining of fatigue and poor sleep and appetite. She has psychomotor retardation and her memory is poor. She is not on antiretroviral therapy. Her CD4 count is 150 cells/ $\mu \mathrm{l}$.

What possible diagnoses should you consider? How would you approach her treatment?

\section{BOX 2}

$\mathrm{Mr} \mathrm{M}$ is a 29-year-old man who was recently diagnosed HIV positive when he presented to health services with a severe upper respiratory tract infection. His CD4 count is 250 cells/ $\mu$ l. He comes for a follow-up appointment in a dishevelled state, smelling of alcohol. He recently lost his job as a clerk in an office, his wife is unemployed and they have a young child. He starts crying in your office and voices suicidal ideation.

What would your immediate management be? And in the longer term?

\section{BOX 3}

Ms B is a 24-year-old woman who was diagnosed HIV positive during antenatal screening. She had PMTCT and her baby was delivered safely 2 weeks ago. She is living with the father of the child, who is aware of her status and has also tested HIV positive. They are both currently asymptomatic. He is very supportive and it is their first child. She is extremely anxious about the well-being and HIV status of her baby. She complains that she is not sleeping and that she gets very irritable with the baby at times. Sometimes she finds herself just bursting into tears for no reason.

What could be going on here? What are the risks in this situation? How would you manage her?

SCREENING FOR COMMON MENTAL DISORDERS IN HIV PRIMARY CARE SERVICES

Because the presentation of depression and anxiety can be unclear and because many people do not volunteer information about substance use disorder, it is critical that people who are infected with HIV should be screened for these disorders. A range of screening instruments have been validated in South Africa and are recommended for use. ${ }^{19-21}$ Some of these are self-report instruments, while others are administered by the health worker. Once an individual is identified through screening, they should receive a more thorough assessment by a trained clinician.

The Substance Abuse and Mental IIIness Symptoms Screener (SAMISS) is a 13-item screening questionnaire developed for use in HIV-positive individuals that can easily be applied in a busy primary care setting (Box 4). The SAMISS was found to have a sensitivity of $86 \%$ and specificity of 95\% in diagnosing DSM-IV-defined substance use and depressive/anxiety disorders, ${ }_{1}^{22}$ and validation of this tool in South Africa is underway. The advantages of using this instrument are that it is reasonably quick to administer and that it covers all the common mental disorders including substance use disorders. 


\section{BOX 4. THE SUBSTANCE ABUSE AND MENTAL ILLNESS SCREENER}

\section{Substance use items:}

1. How often do you have a drink containing alcohol? (Alcoholic drinks include one beer, one glass of wine, a mixed drink of hard liquor, or one wine cooler. Each of these counts as one drink, unless they have double shots, which would equal two drinks.) (If you do not drink, go to question 4.)

0 - Never

1 - Monthly or less

$2-2$ - 4 times a month

$3-2$ - 3 times a week

$4-4$ or more times a week

2. How many drinks do you have on a typical day when you are drinking?

$0-1$ or 2

$1-3$ or 4

$2-5$ or 6

$3-7$ to 9

$4-10$ or more

3. How often do you have four or more drinks on one occasion?

0 - Never

1 - Monthly or less

$2-2$ - 4 times a month

3 - 2 - 3 times a week

$4-4$ or more times a week

4. In the past year, how often did you use non-prescription drugs to get high or change the way you feel?

0 - Never

1 - Monthly or less

$2-2$ - 4 times a month

$3-2$ - 3 times a week

$4-4$ or more times a week

5. In the past year, how often did you use drugs prescribed to you or to someone else to get high or change the way you feel?

0 - Never

1 - Monthly or less

$2-2$ - 4 times a month

3 - 2 - 3 times a week

4- 4 or more times a week

6. In the last year, how often did you drink or use drugs more than you meant to?

0 - Never

1 - Monthly or less

$2-2$ - 4 times a month

$3-2$ - 3 times a week

$4-4$ or more times a week

7. How often did you feel you wanted or needed to cut down on your drinking or drug use in the last year, and not been able to?

0 - Never
1 - Monthly or less

$2-2$ - 4 times a month

3 - 2 - 3 times a week

$4-4$ or more times a week

Patient considered positive for substance use symptoms if any of the following criteria are met:

a) The sum of responses for questions $1-3$ is $\geq 5$

b) The sum of responses for questions $4-5$ is $\geq 3$

c) The sum of responses for questions $6-7$ is $\geq 1$

\section{Mental health items:}

\section{Medications/antidepressants}

8. During the past 12 months, were you ever on medication/antidepressants for depression or nerve problems?

1 - Yes

2 - No

\section{Major depression}

9. During the past 12 months, was there ever a time when you felt sad, blue or depressed for 2 weeks or more in a row?

1 - Yes

2 - No

10. During the past 12 months, was there ever a time lasting 2 weeks or more when you lost interest in most things like hobbies, work, or activities that usually give you pleasure?

1 - Yes

2 - No

\section{Generalised anxiety disorders}

11. During the past 12 months, did you ever have a period lasting 1 month or longer when most of the time you felt worried and anxious?

1 - Yes

2 - No

\section{Panic disorder}

12. During the past 12 months, did you have a spell or an attack when all of a sudden you felt frightened, anxious or very uneasy when most people would not be afraid or anxious?

1 - Yes

2 - No

13. During the past 12 months, did you ever have a spell or an attack when for no reason your heart suddenly started to race, you felt faint, or you couldn't catch your breath? [If respondent volunteers 'only when having a heart attack or due to physical causes', mark 'No'] 1 - Yes

2 - No

Patient considered positive for symptoms of mental illness if he/she responded yes to any mental health question. 
PREVENTION OF COMMON MENTAL DISORDERS IN HIV PRIMARY CARE SERVICES

Given the increased risk of common mental disorders in HIV-infected individuals, it makes sense to put basic preventive services into place in HIV treatment services. These include support groups, counselling, psycho-education, social work and occupational therapy interventions, as available. It is important to note that services of this kind are commonly available as part of HIV primary care services (particularly in the context of antiretroviral therapy), but are usually focused on treatment initiation and adherence; however, such types of psychosocial support have important additional benefits in promoting mental health. Individuals who provide this kind of psychosocial support should be trained to identify patients who may have a common mental disorder (e.g. using the SAMISS), and to refer them for appropriate assessment and intervention. Ideally, psychiatric and mental health care services should be offered on site in HIV treatment services, but this may not always be possible and specialist services are not required to effectively diagnose and manage many common mental disorders. Alternatively, it would be important to identify referral resources and establish good links and communication channels between the HIV treatment service and the psychiatric/mental health services.

\section{MANAGEMENT OF COMMON MENTAL DISORDERS}

Treating common mental disorders has direct benefits on the quality of life and well-being of affected individuals. In addition, there is evidence from international research that untreated depressive and anxiety disorders lead to poor antiretroviral adherence ${ }^{23}$ and may speed HIV disease progression. ${ }^{24-26}$ The management of mental disorders requires a holistic approach with attention to biological as well as psychosocial factors.

\section{BIOLOGICAL MANAGEMENT}

Identify and treat any underlying or associated general medical condition

HIV infection itself is associated with features of depression and apathy. Other chronic conditions such as peripheral neuropathy, or intra-cranial secondary infections or tumours, may produce depression. These include progressive multifocal leuco-encephalopathy or vasculopathy. Usually a careful history, physical examination and routine investigations (e.g. serological testing for syphilis) are adequate to rule these out as causes of psychiatric symptoms, and special investigations (such as lumbar puncture or computed tomography of the brain) are not necessary in many primary care settings.
Assess cognitive impairment

Cognitive impairment often co-exists with depressive disorders, and may be a confounding variable. It is sometimes difficult to assess whether there is depression in the presence of dementia, and depressive illness may also present with cognitive features consistent with dementia. If there are other features and markers of late-stage HIV infection, the need for antiretroviral medication should be considered, as this is an important treatment for HIV-associated dementia (see related article by Singh in this edition).

\section{Assess for suicidality}

Suicidal ideation is common in HIV-infected individuals. In a study in South Africa, ${ }^{27} 22.5 \%$ of a sample of HIV-infected individuals attending HIV treatment sites had experienced suicidal ideation in the past, and $8.6 \%$ had current suicidal ideation; $69 \%$ of the participants with past suicidal ideation had experienced this as a result of their diagnosis. The period after diagnosis is therefore a period of high risk. In the same study, suicidal ideation was strongly associated with the presence of a depressive disorder. It is important to ask patients who are at high risk about thoughts of suicide, as these may not be volunteered. A thorough risk assessment should be conducted, and the need for inpatient admission should always be considered.

Treat depressive or anxiety disorders with medication when necessary

Antidepressant medication is effective in treating both depressive and anxiety disorders in HIV-infected people, even in the presence of cognitive impairment (for more detail on specific medications and their use, see the article in this edition on psychotropic prescribing in HIV-infected individuals). Antidepressant medication can be used safely in combination with antiretroviral medication, as long as one is aware of several specific drug interactions and monitors the patient, adjusting medication when necessary. Generally antidepressant medication should be used for 6 months to 1 year to treat a single episode of a depressive illness. People with recurrent episodes should remain on longterm treatment. Anxiety disorders are best managed with a combination of psychosocial interventions and judicious use of medication. Benzodiazepines should be avoided, only being used in acute crisis situations for short periods of time (less than 2 weeks). Should medication be needed to manage anxiety, the selective serotonin reuptake inhibitors (SSRIs) should be used.

\section{Manage substance withdrawal and provide rehabilitation for substance use disorders}

The initial intervention in terms of substance use disorders is safe medical detoxification, followed by assess- 
ment of the need for and possibilities of rehabilitation. Motivational interviewing is a useful tool for engaging people with substance use disorders in treatment of their addictive behaviour. Rehabilitation usually consists of group and individual psychotherapy, and can usually be managed on an outpatient basis. Support groups, where available, are useful in keeping people sober and drug-free.

\section{INTERPERSONAL, COGNITIVE-BEHAVIOURAL PSYCHOTHERAPY}

There is evidence of benefit from interpersonal psychotherapy for the treatment of depressive disorders in HIV-infected individuals. ${ }^{28}$ Cognitive-behavioural therapy (CBT) has been shown to have benefit in depressive illnesses in general primary health care. ${ }^{28}$ Supportive therapy and support groups are not sufficient where individuals are depressed, but support group facilitators should be trained to identify people who may be depressed. An adaptation of CBT for people living with HIV, called cognitive-behavioural stress management (CBSM), has been found to be effective when conducted in group settings in HIV clinics in the USA. ${ }^{28}$ This intervention includes didactics on physiological effects of stress, stress management strategies, CBT interpretation of stress and emotions (addressing cognitive distortions, automatic thoughts), coping skills training, assertiveness training, anger management, identification of social supports, and group support. This is a structured intervention that could be implemented in HIV clinics or primary care settings where group treatment can be used to treat a number of depressed individuals at the same time, and it deserves attention in South Africa.

Most common mental disorders can be managed effectively in primary care, but there are several specific indications for referring a patient to specialist psychiatric and mental health services (Box 5).

\section{BOX 5}

Indications for referring a patient to specialist psychiatric and mental health services:

1. Any patient who requires inpatient treatment should be referred for a psychiatric assessment.

2. Any patient who presents with suicidal ideation should be referred for a psychiatric assessment.

3. Failure to respond to primary care interventions as outlined above.

4. Patients who fail to respond to an equivalent dose of fluoxetine or citalopram of $20 \mathrm{mg}$ per day for a depressive or anxiety disorder should be referred for a psychiatric assessment or opinion.

\section{DISCUSSION OF CASE SCENARIOS}

Mrs K (Box 1) has stage 3 or 4 HIV disease. She may have neurocognitive impairment and she may also have a depressive disorder. Assessment should include physical examination, and assessment of her cognitive status. She is clearly a candidate for antiretroviral therapy, which should be started as soon as possible. One would re-assess her mental state regularly, and if her mood did not lift once she has recovered physically, one would consider starting her on an SSRI.

A suicide risk assessment should be carried out on Mr M (Box 2). Because he is intoxicated, he would need to be supervised until he became sober. His wife could be called into the clinic to discuss the situation with her. It would be important to educate him about alcohol abuse and its consequences in terms of his HIV status as well as his mood. Another important consideration would be when to start antiretroviral treatment. A social work referral should be made for assistance or advice regarding the couple's financial situation, as well as referral to a support group or a psychologist depending on his condition. His mood should be monitored over time and if it remains low one would consider starting him on an antidepressant.

Ms B (Box 3) could be suffering from postpartum depression. It is important to assess her mental state and do a risk assessment with regard to selfharm as well as possible harm to her baby. One could call her husband in and discuss the situation with them both. If she has features of a depressive disorder, she should be started on an SSRI. It is also important to make sure that there is adequate support and supervision for her and her baby. If the safety of either is a concern, she should be admitted to hospital.

\section{REFERENCES}

1. Patel V, Chisholm D, Rabe-Hesketh S, Dias-Saxena F, Andrew G, Mann A. Efficacy and cost-effectiveness of drug and psychological treatments for common mental disorders in general health care in Goa, India: a randomized controlled trial. Lancet 2003; 361: 33-39.

2. Sumathipala $A$, Hewege $S$, Hanwella R, Mann H. Randomized controlled trial of cognitive behaviour therapy for repeated consultations for medically unexplained complaints: a feasibility study in Sri Lanka. Psychol Med 2000; 30: 747-757.

3. World Health Organization and World Organization of Family Doctors (Wonca). Integrating Mental Health into Primary Care: A Global Perspective. Geneva: WHO Press, 2008.

4. Stein DJ, Seedat $S$, Herman AA, et al. Findings from the First South African Stress and Health Study. Policy brief, October 2007. Tygerberg: South African Medical Research Council, 2007

5. Stein DJ, Seedat $S$, Herman $A A$, et al. Lifetime prevalence of psychiatric disorders in South Africa. BrJ Psychiatry 2008; 192: 112-117.

6. Morrison MF, Pettito JM, Ten Have T, et al. Depressive and anxiety disorders in women with HIV infection. Am J Psychiatry 2002; 159: 789-796.

7. Ciesla GR, Roberts JE. Meta-analysis of the relationship between HIV infection and risk for depressive disorders. Am J Psychiatry 2001; 158: 725-730. 
8. Olley BO, Gxamza F, Seedat $S$, et al. Psychopathology and coping in recently diagnosed HIV/AIDS patients - the role of gender. S Afr Med J 2003; 93: 928931.

9. Olley BO, Seedat $S, N e i D G$, et al. Predictors of major depression in recently diagnosed patients with HIV/AIDS in South Africa. AIDS Patient Care STDs 2004; 18: 481-487.

10. Olley BO, Seedat $S$, Stein DJ. Persistence of psychiatric disorders in a cohort of HIV/AIDS patients in South Africa: A 6-month follow-up study. J Psychosom Res 2006; 61: 479-484

11. Olley BO, Zeier MD, Seedat $\mathrm{S}$, et al. Post-traumatic stress disorder among recently diagnosed patients with HIV/AIDS in South Africa. AIDS Care 2005; 17: 550557.

12. Els C, Boshoff W, Scott C, et al. Psychiatric co-morbidity in South African HIV/ AIDS patients. SAfr Med J 1999; 89: 992-995.

13. Freeman $M$, Nkomo N, Kafaar Z, Kelly K. Factors associated with prevalence of mental disorder in people living with HIV/AIDS in South Africa. AIDS Care 2007; 19: 1201-1209.

14. Martin L, Tummala R, Fernandez F. Psychiatric management of HIV-1 infection and AIDS. Psychiatr Ann 2002; 32: 133-140.

15. Stolar A, Catalano G, Hakala SM, et al. Mood disorders and psychosis in HIV. In: Citron K, Brouillette M-J, Beckett $\mathrm{A}$, eds. HIV and Psychiatry: A Training and Resource Manual. 2nd ed. Cambridge: Cambridge University Press, 2005: 30-55.

16. Blalock AC, Sharma SM, McDaniel JS. Anxiety disorders and HIV disease. In: Citron K, Brouillette M-J, Beckett A, eds. HIV and Psychiatry: A Training and Resource Manual. 2nd ed. Cambridge: Cambridge University Press, 2005: 120127.

17. Koenig HG, George LK, Peterson BL, et al. Depression in medically ill hospitalized older adults: Prevalence, characteristics, and course of symptoms according to six diagnostic schemes. Am J Psychiatry 1997; 154: 1376-1383.

18. Pollak SD. Early adversity and mechanisms of plasticity: Integrating affective neuroscience with developmental approaches to psychopathology. Dev
Psychopathol 2005; 17: 735-752. http://journals.cambridge.org/download (accessed 13 June 2007).

19. Kroenke K, Spitzer RL, Williams JBW. The PGQ-9 validity of a brief depression severity measure. J Intern Med 2001; 16: 606-613.

20. Cherian VI, Peltzer K, Cherian L. The factor structure of the Self Report Questionnaire (SRO20) in South Africa. East Afr Med J 1998; 75(11): 654-656.

21. Babor TF, Higgins-Biddle JC, Saunders JB, Monteiro MG. AUDIT: The Alcohol Use Disorders Identification Test. Guidelines for Use in Primary Care. World Health Organization. Department of Mental Health and Substance Dependence. 2nd ed. Geneva: WHO Press, 2001.

22. Pence BW, Gaynes BN, Whetton $K$, et al. Validation of a brief screening instrument for substance abuse and mental illness in HIV-positive patients. J Acquir Immune Defic Syndr 2005; 40: 434-444.

23. Ickovics JR, Meade CS. Adherence to antiretroviral therapy among patients with HIV: A critical link between behavioral and biomedical sciences. J Acquir Immune Defic Syndr 2002; 31: 98-102.

24. Evans DL, Ten Have TR, Douglas SD, et al. Association of depression with viral load, CD8 lymphocytes, and natural killer cells in women with HIV infection. Am $J$ Psychiatry 2002; 159(10): 1752-1758.

25. Ickovics JR, Hamburger ME, Vlahov D, et al. Mortality, CD4 cell count decline, and depressive symptoms among HIV-seropositive women: Longitudinal analysis from the HIV Epidemiology Research Study. JAMA 2001; 285: 1466-1474. http://0-gateway.ut.ovid.com.innopac.wits.ac.za/gw2/ovidweb.cgi (accessed 25 May 2006).

26. Leserman J. HIV disease progression: Depression, stress and possible mechanisms. Biol Psychiatry 2003; 54: 295-306. http:/www.sobp.org/journal/ (accessed 24 April 2007).

27. Thom RGM. Depressive and anxiety disorders in HIV-infected individuals attending HIV-treatment facilities at various sites in South Africa. PhD thesis, University of the Witwatersrand, December 2008

28. Ferrando SJ, Freyburg Z. Treatment of depression in HIV positive individuals: A critical review. Int Rev Psychiatry 2008; 20(1): 61-71. 